\title{
Optimized software synthesis for digital signal processing algorithms - an evolutionary approach
}

\section{Report}

\section{Author(s):}

Teich, Jürgen; Zitzler, Eckart; Bhattacharyya, Shuvra S.

Publication date:

1998-01

\section{Permanent link:}

https://doi.org/10.3929/ethz-a-004290022

\section{Rights / license:}

In Copyright - Non-Commercial Use Permitted

\section{Originally published in:}

TIK Report 32 


\title{
Optimized Software Synthesis for Digital Signal Processing Algorithms - An Evolutionary Approach
}

\author{
Jürgen Teich and Eckart Zitzler \\ Computer Engineering and Communication Networks Lab (TIK) \\ Swiss Federal Institute of Technology (ETH) \\ Gloriastrasse 35, CH-8092 Zurich \\ Switzerland \\ Shuvra S. Bhattacharyya \\ Department of Electrical Engineering, and \\ Institute for Advanced Computer Studies (UMIACS) \\ University of Maryland \\ College Park MD 20742 \\ U.S.A. \\ TIK-Report \\ Nr. 32, January 1998
}




\section{Contents}

1 Introduction $\quad 1$

1.1 Motivation ........................... 2

1.2 Proposed Approach . . . . . . . . . . . . . . . 4

1.3 Related Work . . . . . . . . . . . . . . 5

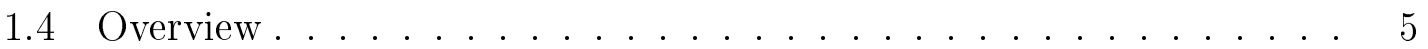

2 An Evolutionary Approach for Memory Optimization 7

2.1 The SDF-scheduling framework . . . . . . . . . . 7

2.1.1 Background and Notation . . . . . . . . . . 7

2.1.2 Code generation model .............. 8

2.1.3 Buffer cost model . . . . . . . . . . . . . . . 9

2.1.4 Buffer Memory Optimization ............ 9

2.2 Why Using an Evolutionary Algorithm . . . . . . . . . . . . 10

2.3 Coupling the Evolutionary Algorithm to the Existing Framework . . . 10

2.3.1 Exploration of topological sorts using the EA . . . . . . 10

2.3.2 Dynamic Programming Post Optimization . . . . . . . . 11

3 Parametrization of the Evolutionary Algorithm 13

3.1 Coding and Repair Mechanism . . . . . . . . . . . . . 14

3.2 Genetic Operators . . . . . . . . . . . . . . 15

3.3 Crossover Probability and Mutation Probability . . . . . . . . 17

4 Experiments 20

4.1 Comparing the Evolutionary Algorithm to APGAN . . . . . . . . 22

4.2 Comparing the Evolutionary Algorithm to RPMC . . . . . . . . . . 24

4.3 Evolutionary Algorithm versus Monte Carlo . . . . . . . . . . . . 25

4.4 Evolutionary Algorithm versus Hill Climbing . . . . . . . . . . . 26

5 Summary and Conclusions $\quad 29$ 


\begin{abstract}
This paper addresses the problem of trading-off between the minimization of program and data memory requirements of single-processor implementations of dataflow programs. Based on the formal model of synchronous data flow (SDF) graphs [LM87], so called single appearance schedules are known to be program-memory optimal. Among these schedules, buffer memory schedules are investigated and explored based on a two-step approach: (1) An Evolutionary Algorithm (EA) is applied to efficiently explore the (in general) exponential search space of actor firing orders. (2) For each order, the buffer costs are evaluated by applying a dynamic programming post-optimization step (GDPPO). This iterative approach is compared to existing heuristics for buffer memory optimization.
\end{abstract}




\section{Chapter 1}

\section{Introduction}

Dataflow specifications are widespread in areas of digital signal and image processing. In dataflow, a specification consists of a directed graph in which the nodes represent computations and the arcs specify the flow of data. A node is allowed to execute (fire) in case a certain firing rule is satisfied. If a node fires, it consumes a certain amount of data from the inputs and produces a certain amount of data on the outputs.

Synchronous dataflow [LM87] is a restricted form of dataflow in which the nodes, called actors have a simple firing rule: The number of data values (tokens, samples) produced and consumed by each actor is fixed and known at compile-time.

Example 1.1 Figure 1.1 shows a simple SDF graph with four actors. A node is enabled for firing in case at least a fixed number of input tokens (as indicated by the arc annotation) has accumulated on each input arc. When the actor executes, a fixed number of output tokens are produced at its outgoing arcs which is also a constant specified at compile-time and annotated at the tail of each outgoing arc.

The major reason why the SDF model is widely used as the underlying specification model are the abilities to express multirate systems, parallelism, and that many important aspects such as deadlock detection and scheduling can be determined at compile-time.

As a matter of fact, the SDF model is used in industrial DSP design tools, e.g., SPW by Cadence, COSSAP by Synopsys, and Advanced Design System from Hewlett-Packard, as well as in research-oriented environments, e.g., [BHLM91; $\mathrm{LEP}^{+}$90; RPM92]. Those systems include code generation tools with code (usually optimized assembly code) stored for each actor in a target-specific library. Typically, code is generated from a given schedule by instantiating actor code in the final program. Subroutine calls may have unacceptable overhead, especially if there are

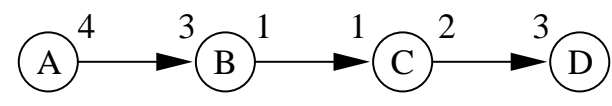

Figure 1.1: A simple SDF graph. 
many small tasks. Hence, a code generation method that generates inline code is generally assumed.

With this model, it is evident that the size of the required program memory strongly depends on the number of times an actor appears in a schedule. So called single appearance schedules, where each actor appears only once (however possibly embedded in a nested loop) in a schedule, are evidently program memory optimal under this model of inline code generation. Results on the existence of such schedules have already been published for general SDF graphs [Bha94]. For acylic graphs, there always exists at least one single appearance schedule.

In this paper, we treat the problem of generating single appearance schedules that minimize the amount of required buffer memory for the class of acyclic SDF graphs. Such a methodology may be considered as part of a general framework that considers general SDF graphs and generates schedules for acyclic subgraphs using our approach. In particular, necessary and sufficient conditions for the existence of single appearance schedules for general SDF graphs and efficient algorithms for computing them have been given in [BBHL95; BL94]. These techniques require decomposing each strongly connnected component into an acyclic graph that consists of clusters of smaller strongly connected components, constructing a single appearance schedule for this acyclic graph, and then recursively applying this procedure to each clustered strongly connected component to obtain the subschedule for the corresponding cluster.

\subsection{Motivation}

Given is an acyclic SDF graph in the following. The number of single appearance schedules that must be investigated is at least equal to (and often much greater than) the number of topological sorts of actors in the graph. Note that this number may be exponential in the size of the graph; e.g., a complete bipartite graph with $2 n$ nodes has $(n !)^{2}$ possible topological sorts. This complexity prevents techniques based on enumeration from being applied sucessfully.

In [BML97], a heuristic called APGAN (for algorithm for pairwise grouping of adjacent nodes (acyclic version)) has been developed that constructs a schedule with the objective to minimize buffer memory. This procedure of low polynomial time complexity has been shown to give optimal results for a certain class of graphs having a regular structure. Also, a complementary procedure called RPMC (for recursive partitioning by minimum cuts) has been proposed that works well on more irregular (e.g., randomly generated) graph structures.

Experiments show that, although being computationally efficient, these heuristics turn out not always to provide the best solutions. Even simple testcases may be constructed where the performance (buffer cost) obtained by applying these heuristics differ from the global minimum by more than $2000 \%$, see graph no. 2 in Example 1.2 .

Example 1.2 We consider two testgraphs and compare different buffer optimization algorithms (see Table 1.2). The first graph with 10 nodes in shown also in Fig. 1.2. 


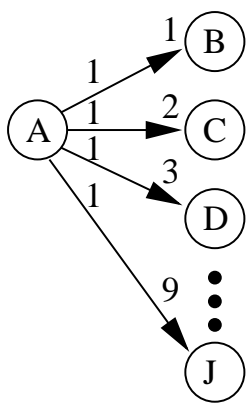

Figure 1.2: A simple SDF graph.

For this rather small and simple graph, already 362880 different topological sorts (actor firing orders) may be constructed. The minimal buffer memory requirement has been evaluated to 3003 whereas for the worst topological sort, the minimal costs were computed as 15705 memory units.

The second graph is a randomly generated graph with 50 nodes. In the table, 4 different methods are compared with respect to the best cost found and the amount of required CPU-time. The first method uses an Evolutionary Algorithm (EA) that performs 3000 fitness calculations, the second is the APGAN heuristic, the third is the RPMC heuristic, the fourth is a Monte Carlo simulation (3000 random tries) and the fifth an exhaustive search procedure which did not terminate in the second case.

\begin{tabular}{|l||l|c|c|}
\hline Graph \# & method & best cost (units) & runtime (s) \\
\hline 1 & EA & 3003 & 4.57 \\
1 & APGAN & 3015 & 0.02 \\
1 & RPMC & 3151 & 0.03 \\
1 & Monte Carlo & 3014 & 3.3 \\
1 & Exhaust. Search & 3003 & 373 \\
\hline 2 & EA & 669380 & 527.87 \\
2 & APGAN & 15063956 & 1.88 \\
2 & RPMC & 2378112 & 2.03 \\
2 & Monte Carlo & 2600349 & 340.66 \\
2 & Exhaust. Search & $?$ & $?$ \\
\hline
\end{tabular}

Table 1.1: Analysis of existing heuristics on simple testgraphs. The run-times were measured on a SUN SPARC 20.

The motivation of the following work was to develop a methodology that has the following features:

- Cost-competitiveness: the optimization procedure should provide solutions which provide the same or lower buffering costs as the heuristics APGAN and RPMC in most investigated test cases. 


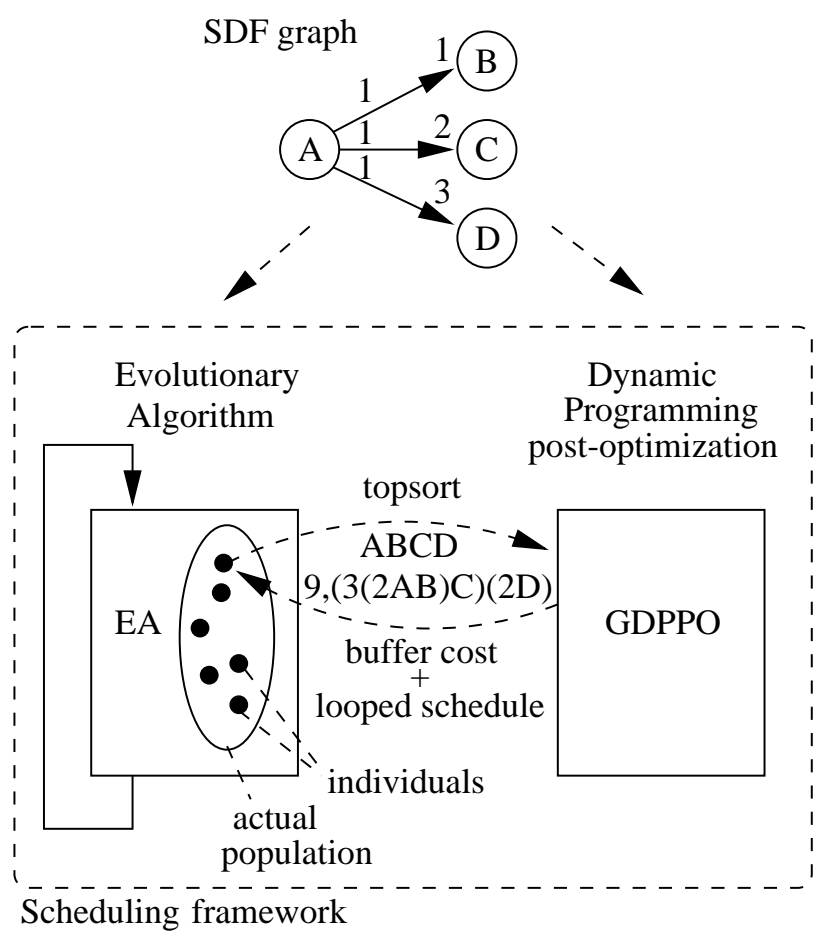

Figure 1.3: Overview of the scheduling framework using Evolutionary Algorithms and Dynamic Programming (GDPPO: generalized dynamic programming post optimization for optimally parenthesizing actor orderings [MBL94], discussed further in Section 2.3.2) for constructing buffer memory optimal schedules.

- Run-time tolerability: in embedded DSP applications, compilers are allowed to spend more time for optimization of code as in general-purpose compilers, because code-optimality is critical [Me95]. Hence, compilation times in the range from 1s to 100s, sometimes even in the order of minutes, still seem to be tolerable.

Therefore, it does not seem reasonable to compare different algorithms in terms of the quality (buffer memory cost) obtained during the run-time limit of the fastest heuristic. Instead, we compare the quality obtained during a tolerable run-time limit, say, e.g., one minute of CPU-time.

\section{$1.2 \quad$ Proposed Approach}

Here, we use a unique two-step approach to find buffer-minimal schedules:

- An Evolutionary Algorithm (EA) is used to efficiently explore the space of topological sorts of actors given an SDF graph using a population of $N$ individuals where each individual of a population encodes a topological sort. 
- For each topological sort, a buffer optimal schedule is constructed based on a well-known dynamic programming post optimization step [MBL94] that determines a loop nest by parenthesization (see Fig. 1.3) that is buffer cost optimal (for the given topological order of actors). The run-time of this optimization step is $\mathcal{O}\left(N^{3}\right)$.

Example 1.3 The overall picture of the scheduling framework is depicted in Fig. 1.3. The approach is called GASAS (for Genetic Algorithm exploration of Single Appearance Schedules). The EA iteratively transforms a population of individuals, each of which is coding a topological sort of the actors of a given SDF graph. The buffer cost of each individual is evaluated by calling a Dynamic Programming post-optimizer that performs a parenthesization of the proposed actor firing order to obtain a buffer-optimal schedule (for the given order of actors). The evaluated optimal costs are returned to the EA that applies transformations to the individuals in order to find better topological sorts. Details on the optimization procedure and the cost function will be explained in the following chapter. The total run-time of the algorithm is $\mathcal{O}\left(Z N^{3}\right)$ where $Z$ is the number of evocations of the dynamic program post-optimizer.

\subsection{Related Work}

The interaction between instruction scheduling and register allocation in procedural language compilers has been studied extensively [Hsu87; ASU86], and optimal management of this interaction has been shown to be intractable [GJ79]. More recently, the issue of optimal storage allocation has been examined in the context of high-level synthesis for iterative DSP programs [DP96], and code generation for embedded processors that have highly irregular instruction formats and register sets [Me95; KNDK96]. However, because of their focus on fine-grain scheduling, the above efforts apply to a homogeneous data flow model - that is, a model in which each computation (dataflow vertex) produces and consumes a single value to/from each incident edge. In particular these efforts do not address the challenges of keeping code size costs manageable in general SDF graphs, in which actor production and consumption parameters may be arbitrary.

Similarly, Fabri [Fab82] and others have examined the problem of managing pools of logical buffers that have varying sizes, given a set of buffer lifetimes, but such efforts are also in isolation of the scheduling problems that we face in the context of general SDF graphs.

\subsection{Overview}

We conclude this chapter with a summary of what follows.

In Chapter 2, the required notation is introduced and the overall methodology is explained in more detail including an explanation of why an Evolutionary Algorithm 
is favorable for design space exploration. Also, the cost model for buffer cost and the role of the dynamic program post-optimizer are outlined.

Chapter 3 explains the Evolutionary Algorithm approach. In particular, we describe how topological sorts for SDF graphs may be efficiently generated and explored. Details on the chosen genetic operators and the coding mechanism are provided.

Finally, a quantitative comparison of this new approach with existing algorithms like APGAN and RPMC [BML97] as well as with other probabilistic optimization methods like Monte Carlo and Hill Climbing are described in Chapter 4. In case a run-time limit of 100 seconds for the exploration of the search space is allowed, the Evolutionary Algorithm approach beats existing heuristics in almost $100 \%$ of the test cases which have been chosen from a library of existing problems and a set of randomly generated test graphs. 


\section{Chapter 2}

\section{An Evolutionary Approach for Memory Optimization}

\subsection{The SDF-scheduling framework}

\subsubsection{Background and Notation}

Synchronous dataflow [LM87] is a restricted form of data flow in which nodes of a directed graph represent actors, consume a fixed amount of data items (tokens, samples) per invocation and produce a fixed amount of output samples per invocation.

Definition 2.1 (SDF graph) An SDF graph $G$ denotes a 5-tuple $G=$ ( $V, A$, produced, consumed, delay) where

- $V$ is the set of nodes (actors) $\left(V=\left\{v_{1}, v_{2}, \cdots, v_{\mathrm{K}}\right\}\right)$.

- A is the set of directed arcs. With source $(\alpha)(\operatorname{sink}(\alpha))$, we denote the source node (target node) of an arc $\alpha \in A$.

- produced $: A \rightarrow \mathbf{N}$ denotes a function that assigns to each directed arc $\alpha \in A$ the number of produced tokens produced $(\alpha)$ per invocation of actor source $(\alpha)$.

- consumed $: A \rightarrow \mathbf{N}$ denotes a function that assigns to each directed arc $\alpha \in A$ the number of consumed tokens per invocation of actor $\operatorname{sink}(\alpha)$.

- delay : $A \rightarrow \mathbf{N}_{0}$ denotes the function that assigns to each arc $\alpha \in A$ the number of initial tokens delay $(\alpha)$.

A schedule is a sequence of actor firings. A properly-constructed SDF graph is compiled by first constructing a finite schedule $S$ that fires each actor at least once, does not deadlock, and produces no net change in the number of tokens queues associated with each arc. When such a schedule is repeated infinitely, we call the resulting infinite sequence of actor firings a valid periodic schedule, or simply valid schedule. 
SDF graphs for which valid schedules exist are called consistent graphs. Systematic techniques exist to efficiently determine whether or not a given SDF graph is consistent and to compute the minimum number of times that each actor must execute in the body of a valid schedule [LM87]. We represent these minimum numbers of firings by a function $q_{\mathrm{G}}$ or simply $q$ in case $G$ is known from the context with $q: V \rightarrow \mathbf{N}$.

Example 2.1 Figure 1.1 shows an example of an SDF graph with four nodes and two edges. The four nodes (actors) are labeled $A, B, C, D$, respectively. The graph is consistent, because there exists a (non-zero) finite actor firing sequence such that the initial token configuration is obtained again. The minimal number of actor firings is obtained as $q(A)=9, q(B)=q(C)=12, q(D)=8$. The schedule

$$
(\infty(2 A B C) D A B C D B C(2 A B C D) A(2 B C)(2 A B C) A(2 B C D))
$$

represents a valid schedule for the SDF graph shown in Fig. 1.1. Here, a parenthesized term ( $\left.n S_{1} S_{2} \cdots, S_{k}\right)$ specifies $n$ sucessive firings of the "subschedule" $S_{1} S_{2} \cdots S_{k}$.

Each parenthesized term $\left(n S_{1} S_{2} \cdots S_{k}\right)$ is referred to as schedule loop having iteration count $n$ and iterands $S_{1}, S_{2}, \cdots, S_{k}$. We say that a schedule for an SDF graph is a looped schedule if it contains zero or more schedule loops.

A schedule is called single appearance schedule if it contains only one appearance of each actor.

Example 2.2 The schedule $(\infty(9 A)(12 B)(12 C)(8 D))$ is a valid single appearance schedule for the graph shown in Fig. 1.1.

In general, a schedule of the form

$$
\left(\infty\left(q\left(N_{1}\right) N_{1}\right)\left(q\left(N_{2}\right) N_{2}\right) \cdots\left(q\left(N_{\mathrm{K}}\right) N_{\mathrm{K}}\right)\right)
$$

where $N_{i}$ denotes the (label of the) $i$ th node of a given SDF graph, and K denotes the number of nodes of the given graph, is called flat single appearance schedule.

\subsubsection{Code generation model}

We consider the problem of code generation by code inlining given a SDF graph specification while considering single processor implementations: Corresponding to each actor in a valid schedule $S$, we insert a code block that is obtained from a library of predefined actors, and the resulting sequence of code blocks is encapsulated within an infinite loop to generate a software implementation. Each schedule loop thereby is translated into a loop in the target code.

Implied by this model of code generation, any valid single appearance schedule gives the minimum code space (program memory) cost. This approximation, however, neglects loop overhead. In practical SDF models of applications, actors are usually DSP subsystems of medium to large granularity, and the code size overhead of a loop is typically small compared to the size of individual code blocks. Thus, neglecting loop overhead does not lead to misleading results in our context. 


\subsubsection{Buffer cost model}

The simplest model for buffering (data memory) is to assume that a distinct area of memory is allocated for each arc of a given graph. In order to determine the amount of data needed to store the tokens that accumulate on each arc during the evolution of a schedule $S$, we define the cost function

$$
\text { buffer_memory }(S)=\sum_{\alpha \in A} \text { max_tokens }(\alpha, S) .
$$

Here, max_tokens $(\alpha, S)$ denotes the maximum number of tokens that accumulate on $\operatorname{arc} \alpha$ during the execution of schedule $S$.

Example 2.3 Consider the schedule $(\infty(9 A)(12 B)(12 C)(8 D))$ for the SDF graph shown in Fig. 1.1. This schedule has a buffer memory requirement of $36+12+24=$ 72. Similarly, the buffer memory requirement of the schedule $(\infty(3(3 A)(4 B))(4(3 C)$ $(2 D))$ ) is $12+12+6=30$.

Another model for buffering is to use a shared buffer of size

$$
\max _{i=1}^{\mathrm{K}-1}\left(q\left(N_{i}\right) \text { produced }\left(N_{i}\right)\right) \text {, }
$$

which gives the maximum amount of data transferred on any arc in one period (one iteration of the outermost loop) of the flat single appearance schedule. In case of nested loops, however, the use of shared buffers may be awkward. Also, the management of pointers and the handling of initial delays on arcs require special attention. In the latter case, there is often no logical place in the buffer to place the delays since the entire buffer might be written over by the time we reach the actor that consumes the delays. Therefore, we will use the non-shared buffer model for all arcs with non-zero initial delays, and decide on the usefulness of buffer sharing for arcs without delays.

\subsubsection{Buffer Memory Optimization}

The following lower bounds on the buffer memory requirements have been published in [BML97]:

Definition 2.2 (Buffer memory lower bound) The buffer memory lower bound (BMLB) of an $S D F$ arc $\alpha \in A$, denoted $B M L B(\alpha)$, is given by

$$
B M L B(\alpha)=\left\{\begin{array}{rll}
(\eta(\alpha)+\operatorname{delay}(\alpha)) & \text { if } & (\operatorname{delay}(\alpha)<\eta(\alpha)) \\
\operatorname{delay}(\alpha) & \text { if } & (\operatorname{delay}(\alpha) \geq \eta(\alpha))
\end{array}\right.
$$

where

$$
\eta(\alpha)=\frac{\operatorname{produced}(\alpha) \text { consumed }(\alpha)}{\operatorname{gcd}(\{\operatorname{produced}(\alpha), \text { consumed }(\alpha)\})} .
$$

If $G$ is an SDF graph, then

$$
\left(\sum_{\alpha \in A} B M L B(\alpha)\right)
$$


is called the BMLB of $G$, and a valid single appearance schedule $S$ for $G$ that satisfies max_tokens $(\alpha, S)=B M L B(\alpha) \forall \alpha \in A$ is called BMLB schedule for $G$.

It should be noted that not all SDF graphs have valid BMLB schedules, but many practical graphs do.

\subsection{Why Using an Evolutionary Algorithm}

From Example 1.2, it became clear that there exist simple graphs for which there is a big gap between the quality of solution obtained using different heuristics such as APGAN and an Evolutionary Algorithm (EA).

Also, the example has shown that an Evolutionary Algorithm for efficiently exploring the vast search space of topological sorts has provided much better solutions than just performing a random search (Monte-Carlo simulation). If the run-time of such an iterative approach is still affordable, a performance gap of several orders of magnitude may be avoided.

Moreover, an Evolutionary Algorithm to efficiently explore the search space seems to be the choice at hand because of the following reasons:

- Topological sorts may be easily coded using an Evolutionary Algorithm. Details on the coding scheme will be given in the following chapter.

- Evolutionary algorithms perform a parallel sampling of the search space by working on populations of individuals.

- The optimization function is allowed to be non-linear and arbitrary complex.

\subsection{Coupling the Evolutionary Algorithm to the Existing Framework}

In this section, we describe the sharing of work for exploring the search space of buffer memory optimal single appearance schedule solutions.

The separation of work has already been figured out in Fig. 1.3.

\subsubsection{Exploration of topological sorts using the EA}

Here, given an acyclic SDF graph, the main difficulties consist in finding a coding of feasible topological sorts. One could use a coding scheme which represents a permutation of the actor set. However, by genetic mutation and crossover, the permutations in general would not represent topological sorts. Penalty functions that punish infeasible permutations would not prevent the needle-in-the-haystack search in some cases. Hence, a simple repair mechanism must be used in order to guarantee each individual in the actual population to represent a topological sort.

Also, the simple extension to allow also permutations where each actor may fire enough times without being in topological order are possible. This may happen 
in case enough delays are accumulated on corresponding input arcs of an actor in question.

Details on the coding scheme are given in the following chapter that deals with all implementation issues of the evolutionary search procedure. Instead, we conclude the chapter by summarizing the way, a provably buffer-memory optimal schedule may be found for a given actor ordering by dynamic programming.

\subsubsection{Dynamic Programming Post Optimization}

Given a consistent acyclic SDF graph $G$ and, for simplicity, assume that each arc $\alpha \in A$ satisfies delay $(\alpha)=0$ (delayless arcs).

In [MBL94], it has been shown that given a topological sort of actors of a consistent, delayless and acyclic SDF graph, a minimum buffer memory schedule over all single-appearance schedules for this graph with the same lexical ordering as the topological sort may be determined as the solution of a dynamic programming problem (DPPO). ${ }^{1}$

Example 2.4 Consider again the SDF graph in Fig. 1.1. With $q(A)=9, q(B)=$ $q(C)=12$, and $q(D)=8$, an optimal schedule is $(\infty(3(3 A)(4 B))(4(3 C)(2 D)))$ with a buffer cost of 30. Given the topological order of nodes $A, B, C, D$ as imposed by the arcs of $G$, this schedule is obtained by parenthesization of the string. Note that this optimal schedule contains a break in the chain at some actor $k, 1 \leq k \leq \mathrm{K}-1$. Because the parenthesization is optimal, the chains to the left of $k$ and to the right of $k$ must also be parenthesized optimally. This structure of the optimization problem is essential for dynamic programming.

Let $b[i, j], 1 \leq i \leq j \leq \mathrm{K}$ denote the minimum buffer cost (over all valid single appearance schedules with the same actor ordering) for scheduling the subgraph induced by the actors $v_{i}, v_{i+1}, \cdots, v_{j}{ }^{2}$

For $1 \leq i \leq j<\mathrm{K}, b[i, j]$ may be calculated as

$$
b[i, j]=\min _{i \leq k<j}\left\{b[i, k]+b[k+1, j]+c_{i, j}[k]\right\},
$$

where $b[i, k]$ is the minimum buffer cost for the subgraph induced by $\left\{v_{i}, \cdots, v_{k}\right\}$ and $b[k+1, j]$ is the minimum buffer cost for the subgraph induced by $\left\{v_{k+1}, \cdots, v_{j}\right\}$, $b[i, i]=0$, and $c_{i, j}[k]$ denotes the memory cost at the split if we split the graph at actor $v_{k}$ into a parenthesized left subgraph induced by $\left\{v_{i}, \cdots, v_{k}\right\}$ and a paren-

\footnotetext{
${ }^{1}$ The extension GDPPO in [BML96] guarantees that given any (not necessarily delayless) consistent SDF graph and a lexical ordering (not necessarily a topological sort), a single appearance schedule is computed that minimizes the buffer memory over all single appearance schedules that have the given lexical ordering (assuming that at least one valid single appearance schedule exists that has the given lexical ordering).

${ }^{2}$ This graph has the node set $V^{\prime}=\left\{v_{i}, v_{i+1}, \cdots, v_{j}\right\}$ and the arc set $A^{\prime}=\{\alpha \in A \mid \operatorname{source}(\alpha) \in$ $V^{\prime}$ and $\left.\operatorname{sink}(\alpha) \in V^{\prime}\right\}$.
} 
thesized right subgraph induced by $\left\{v_{k+1}, \cdots, v_{j}\right\}$. The minimal buffer cost of the complete graph is then obtained as $b[1, \mathrm{~K}] .^{3}$

The split $\operatorname{cost} c_{i, j}[k]$ is obtained as

$$
c_{i, j}[k]=\frac{\sum_{\alpha \in A \cap A_{\text {cut }}}(\operatorname{produced}(\alpha) q(\operatorname{source}(\alpha))}{\operatorname{gcd}\left(\left\{q\left(v_{i}\right), q\left(v_{i+1}\right), \cdots, q\left(v_{j}\right)\right\}\right)}
$$

where $A_{c u t}$ denotes the arc set

$$
A_{\text {cut }}=\left\{\beta \in A \mid\left(\operatorname{source}(\beta) \in\left\{v_{i}, v_{i+1}, \cdots, v_{k}\right\} \text { and } \operatorname{sink}(\beta) \in\left\{v_{k+1}, v_{k+2}, \cdots, v_{j}\right\}\right)\right\}
$$

An easy extension to include also shared buffering is to assume that there is one shared buffer of size

$$
c s_{i, j}=\frac{\max _{i \leq k<j}\left(\sum_{\alpha \in A \cap A_{c u t}}(\operatorname{produced}(\alpha) q(\operatorname{source}(\alpha)))\right.}{\operatorname{gcd}\left(\left\{q\left(v_{i}\right), q\left(v_{i+1}\right), \cdots, q\left(v_{j}\right)\right\}\right)}
$$

for the subgraph with nodes $\left\{v_{i}, v_{i+1}, \cdots, v_{j}\right\}$. Finally, the cost $b[i, j]$ is replaced by $b^{\prime}[i, j]$ with

$$
b^{\prime}[i, j]=\min \left(\left\{b[i, j], c s_{i, j}\right\}\right)
$$

In this case, the optimization procedure also determines which arcs to be implemented in shared buffer and which arcs should be implemented separately.

An extension to include also initial delay on arcs is also possible.

\footnotetext{
${ }^{3}$ In the case of general acyclic graphs with multiple source and/or multiple sink nodes, a dummy node $v_{0}$ that becomes the unique source of all former source nodes may be introduced. For multiple sinks, a dummy node $n_{\mathrm{K}+1}$ may be similarly introduced that becomes target of all former sink nodes. Finally compute the minimum buffer cost as $b[0, \mathrm{~K}+1]$.
} 


\section{Chapter 3}

\section{Parametrization of the Evolutionary Algorithm}

As described in the foregoing chapter, we use an Evolutionary Algorithm to sample the search space of all possible topological sorts of a given SDF graph. Each topological sort is assigned a fitness value, reflecting buffer memory requirements, which is computed by the SDF framework. The aim is to find the graph node order which yields a single appearence schedule with minimal buffer cost among all possible orders.

The flowchart of the Evolutionary Algorithm is depicted in Figure 3.1. The left hand side shows the single steps of the algorithm, the right hand side visualizes the effect of each step on the population. The contents of the population are exemplary and refer to the simple SDF graph presented in Figure 1.1.

In the first step an initial population is created. It contains randomly generated individuals, the phenotype of which represents a topological sort. The evolution of new generations is done in the main loop. After the fitness evaluation, the population is modified by applying the three genetic operators selection, recombination, and mutation. While the selection operator increases the average quality of the population, the two variation methods recombination and mutation serve the exploration of new topological sorts. In contrast to other descriptions of Evolutionary Algorithms [Gol89][Koz92], here we view selection, recombination, and mutation as independent processes subsequently working on the whole population.

In the following, we deal with the details of the Evolutionary Algorithm. The problem of encoding node orders and transforming arbitrary orders into valid topological sorts is addressed in Section 3.1. Section 3.2 treats the kind of genetic operators we have implemented, while section 3.3 examines the influence of different crossover and mutation probabilities on the performance of the Evolutionary Algorithm. 


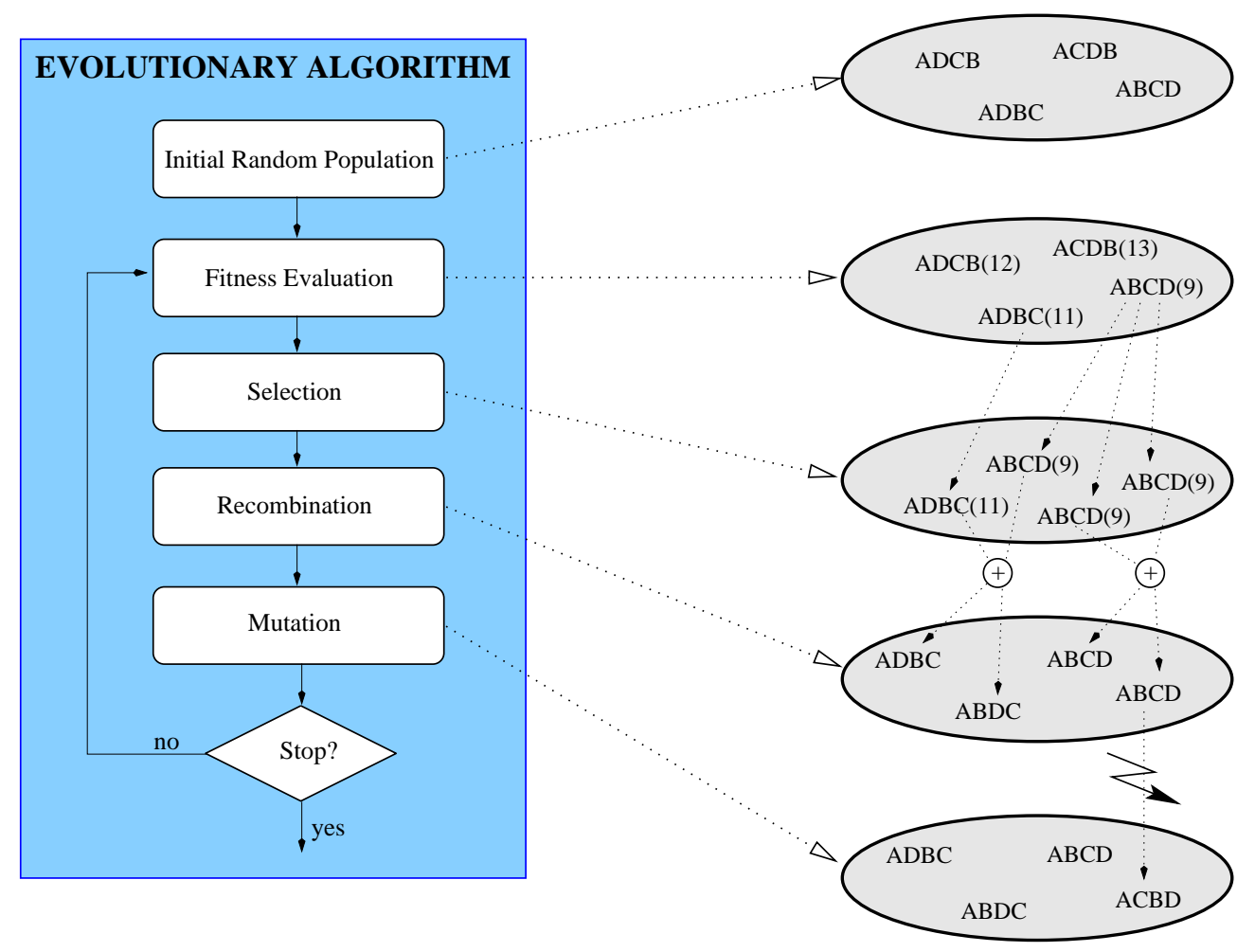

Figure 3.1: Flowchart of the Evolutionary Algorithm.

\subsection{Coding and Repair Mechanism}

Our problem is a combinatorial optimization problem rather than a numerical optimization problem. It naturally suggests to use an order-based representation. But we do not consider arbitrary orderings of the nodes in the graph because we are only interested in topological sorts. Instead, each individual encodes a permutation over the set of nodes. Hence, the initial population consists of randomly created permutations.

Beyond it, a simple repair mechanism, transforming permutations into topological sorts, guarantees that every genotype can be mapped to a valid topological sort. So, there are no infeasible individuals in the population. On the other hand, since each topological sort is simultaneously a permutation, the whole search space is covered by this representation.

The skeleton of the repair algorithm is formed by a common algorithm for sorting graphs topologically (see Figure 3.2). In each step, a node with an indegree equal to zero is chosen and removed from the graph (together with the incident edges). The order in which the nodes appear determines the topological sort. With it, the tie between several nodes with no ingoing edges is normally broken by random. Our algorithm, however, always selects the node at the leftmost position within the permutation. This ensures on the one hand, that each individual is mapped unambiguously to one topological sort, and, on the other hand, that every topological 


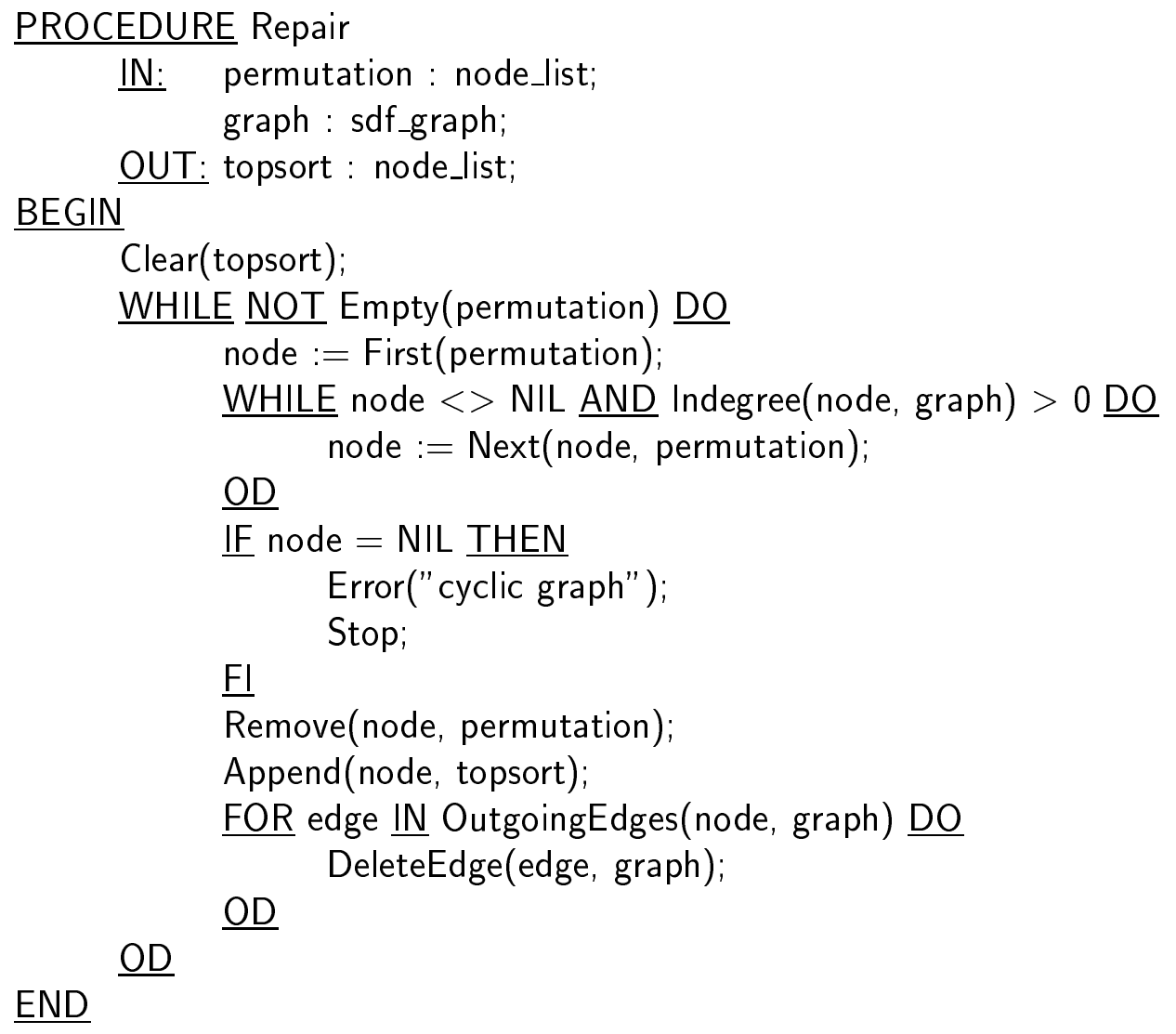

Figure 3.2: Repair algorithm for mapping permutations to topological sorts.

sort has at least one encoding.

Example 3.1 Recall the SDF graph depicted in Figure 1.2, and suppose, the repair algorithm is working on the permutation BCDEFAGHIJ. Since the node A has no ingoing edges but is predecessor of all other nodes, it has to be placed first in any topological sort. The order of the remaining nodes is unchanged. Therefore, the resulting topological sort after the repair procedure in Figure 3.2 is ABCDEFGHIJ.

Note that in the real system an optimized algorithm has been implemented which avoids delete operations on the SDF graph.

\subsection{Genetic Operators}

Many selection schemes exist as well as crossover and mutation methods specialized for order-based representations. In the following, we outline the operators we have chosen. 
Selection The selection scheme chosen is tournament selection. In tournament selection a fixed number of individuals is picked out randomly, and the individual having the best fitness value (lowest buffer cost) within this group is copied to the new population. This process is repeated until the new population has been filled up.

The advantage of this algorithm is its linear time complexity $\mathcal{O}(N)$, where $N$ denotes the population size. Fitness proportionate selection has linear time complexity too, but in contrast to tournament selection it is not translation invariant [dlMT93]. That means increasing the fitness values by adding a constant causes a change in the result of the selection process. ${ }^{1}$ On the other hand, e.g., rank selection, which is translation invariant, needs al least $\mathcal{O}(N \log N)$ run-time due to sorting the population.

The issue of run-time is important for our concerns. Although in Section 1.1, run-time tolerability was claimed as an essential feature of our methodology, we paid special attention to a fast implementation of the Evolutionary Algorithm in order to be competitive to the existing, deterministic heuristics.

Independent of this selection scheme, an elitist strategy has been implemented: the best individual per generation is preserved by simply copying it to the population of the next generation.

Crossover Since individuals encode permutations, it is crucial that the variation operators do not destroy the permutation property. Each node has to appear exactly one time in a sequence coded by a chromosome. To accomplish this task, the uniform order-based crossover operator [Dav91][FM91] is applied. We give a short description of it below.

1) select parents

2) generate random bit string

3) fill in selected positions

4) collect missing elements in list

5) re-order list

6) fill up children

parent 1

A B C D E F G H I

parent 2

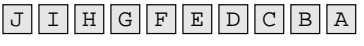

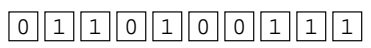

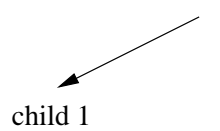

child 1
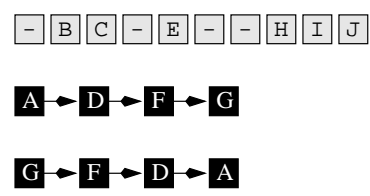

G B C F E D A H I I
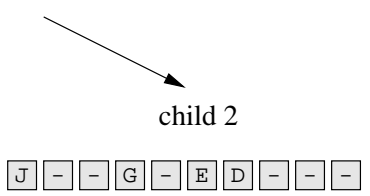

$\mathbf{I}-\mathbf{B}-\mathbf{F}-\mathbf{C}-\mathbf{B}-\mathbf{A}$ $A \rightarrow B \rightarrow C-F \rightarrow B \rightarrow I$

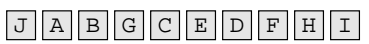

Figure 3.3: Uniform oder-based crossover.

\footnotetext{
${ }^{1}$ Since the buffer costs may vary extremely for different applications, translation invariance is an important aspect.
} 
First, a bit string is randomly generated that is the same length as the parents. The bit positions correspond to the positions within the permutations defined by the parents. Then the first child is partly filled up. The node annotations of the first parent are copied to this child at the positions where the bit string contains a "1". Analogously, the second child inherits the node annotations of the second parent wherever a " 0 " occurs. Now, both children have gaps at complementary positions. In a third step, for each child a list is built up which contains all nodes not yet specified in the child. Afterwards, the list of the first child is sorted according to the node order in the second parent. Again, the same is done to the list of the second child. The relative order of the nodes in the list is identical to their relative order in the first parent. Finally, step by step and from left to right, the list elements are inserted at the gaps of the corresponding child.

Example 3.2 Let the first parent be the sequence ABCDEFGHI and the second parent the same sequence in reverse order. The intermediate results of the crossover phase are shown in Figure 3.3. At the end, two children, GBCFEDAHIJ and JABGCEDFHI, have been created.

Mutation Mutation is done by permuting the elements between two selected positions, whereas both the positions and the subpermutation are chosen by random. That is what Davis calls scramble sublist mutation [Dav91].

Example 3.3 Figure shows an example for this operator. The chromosome ABCDEFGHIJ mutates to the sequence ABCFDGEHIJ.

\author{
1) select individual \\ 2) choose two random positions \\ 3) permutate sublist randomly
}

A B C D E F G H I J
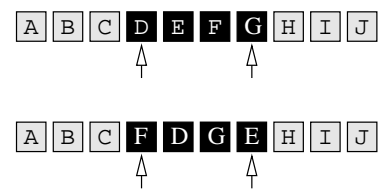

Figure 3.4: Scramble sublist mutation.

\title{
3.3 Crossover Probability and Mutation Proba- bility
}

To the recombination operator as well as to the mutation operator, probabilities for their application are associated, namely the crossover probability $p_{c}$ and the mutation probability $p_{m}$. The setting of these two parameters might have great influence on the outcome and the convergence speed of the Evolutionary Algorithm. 
Therefore we tried several different $p_{c}-p_{m}$-combinations on a few random graphs containing 50 nodes. $^{2}$

Based on experimental results (not to go further into detail), we have chosen a population size of 30 individuals. The crossover rates we tested are $0,0.2,0.4,0.6$, and 0.8 , while the mutation rates cover the range from 0 to 0.4 by a step size of 0.1 . Altogether, the Evolutionary Algorithm ran with 24 various $p_{c}-p_{m}$-settings on every test graph. It stopped after 3000 fitness evaluations. For each combination we took the average fitness (buffer cost) over ten independent runs.

Exemplary, the results for a particular graph are visualized by the 3D plot in Figure 3.5; the results for the other random test graphs look similar.

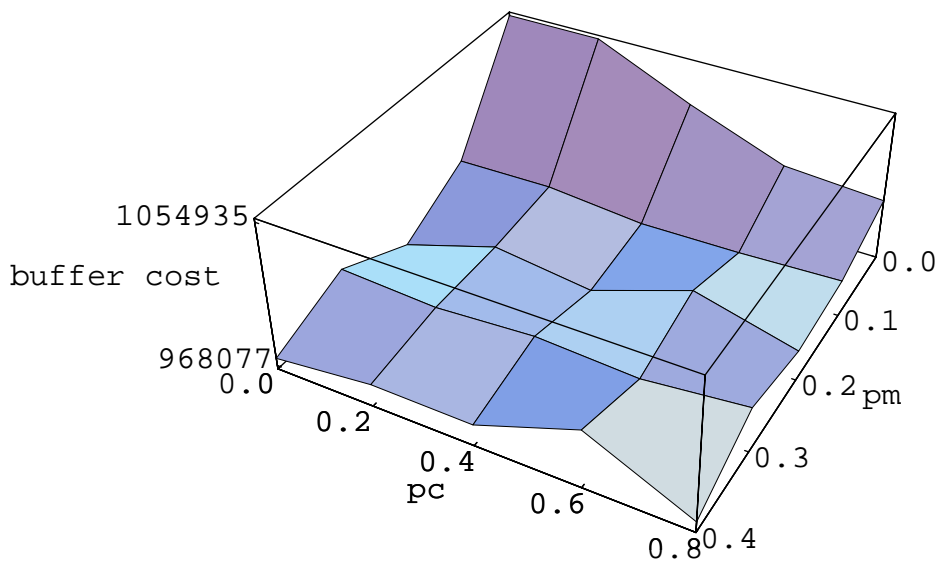

Figure 3.5: Influence of the crossover probability $p_{c}$ and the mutation probability $p_{m}$ on the average fitness for a particular test graph (3000 fitness evaluations).

Obviously, mutation is essential to this problem. Setting $p_{m}$ to 0 leads to the worst results of all probabilty combinations. If $p_{m}$ is greater than 0 , the obtained average buffer costs are significantly smaller - almost independently of the choice of $p_{c}$. As can be seen in Figure 3.6 this is due to premature convergence. The curve representing the performance for $p_{c}=0.2$ and $p_{m}=0$ goes horizontally after about 100 fitness evaluations. No new points in the search space are explored. As a consequence, the Monte Carlo optimization method, that simply generates random points in the search space and memorizes the best solution, might be a better approach to this problem. We investigate this issue in Chapter 4 .

On the other hand, the impact of the crossover operator on the overall performance is not as great as that of the mutation operator. With no mutation at all, increasing $p_{c}$ yields decreased average buffer cost. But this is not the same to cases where $p_{m}>0$. The curve for $p_{c}=0.6$ and $p_{m}=0.2$ in Figure 3.6 bears out this observation. Beyond it, for this particular test graph a mutation probability of $p_{m}=0.2$ and a crossover probability of $p_{c}=0$ leads to best performance. This might

\footnotetext{
${ }^{2}$ Graphs consisting of less nodes are not very well suited to obtain reliable values for $p_{c}$ and $p_{m}$, because the optimum is yet reached after a few generations, in most cases.
} 
be interpreted as hint that Hill Climbing is also suitable in this domain. The Hill Climbing approach generates new points in the search space by applying a neighborhood function to the best point found so far. A comparison of the Evolutionary Algorithm to Hill Climbing is presented in chapter 4.

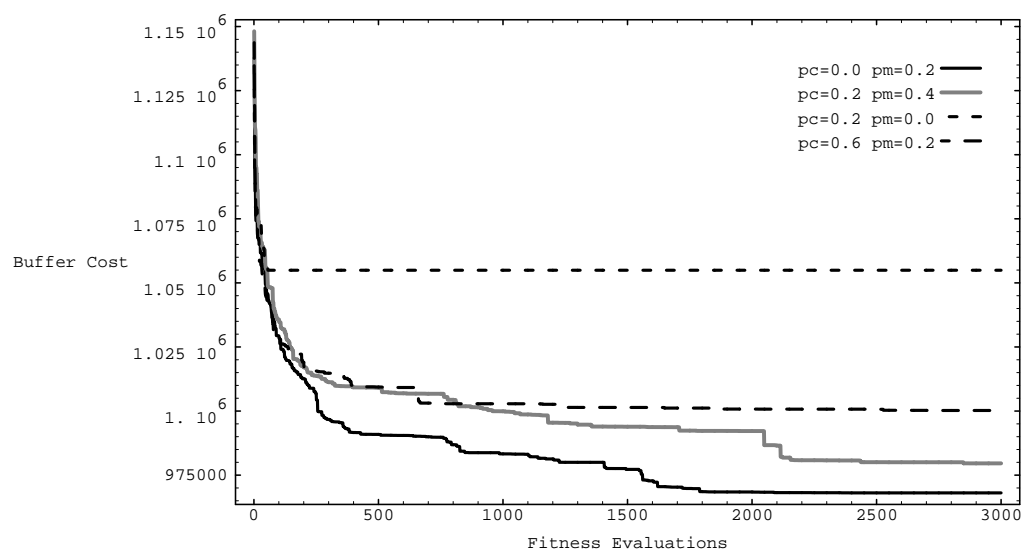

Figure 3.6: Performance of the Evolutionary Algorithm according to four different $p_{c}-p_{m}$-combinations; each graph represents the average of ten runs.

Nevertheless, with respect to the results on other test graphs, we found a crossover rate of $p_{c}=0.2$ and a mutation rate of $p_{m}=0.4$ to be most appropriate for this problem. 


\section{Chapter 4}

\section{Experiments}

To evaluate the performance of the Evolutionary Algorithm we tested it on several practical examples of acyclic, multirate SDF graphs as well as on 200 acyclic random graphs, each containing 50 nodes and having 100 edges in average. The obtained results were compaired against the outcomes produced by APGAN, RPMC, Monte Carlo (MC), and Hill Climbing (HC). We also tried a slightly modified version of the Evolutionary Algorithm which first runs APGAN and then inserts the computed topological sort into the initial population.

Table 4.1 shows the results of applying GDPPO to the schedules generated by the various heuristics on several practical SDF graphs; the satellite receiver example is taken from [RWM95], whereas the other examples are the same as considered in [BML96]. The probabilistic algorithms ran once on each graph and were aborted after 3000 fitness evaluations. Additionally, an exhaustive search with a maximum run-time of 1 hour was carried out; as it only completed in two cases ${ }^{1}$, the search spaces of these problems seem to be rather complex.

In all of the practical benchmark examples that make up Table 4.1 the results achieved by the Evolutionary Algorithm equal or surpass the ones generated by RPMC. Compared to APGAN on these practical examples, the Evolutionary Algorithm is neither inferior nor superior; it shows both better and worse performance in two cases each. Furthermore, the performance of the Hill Climbing approach is almost identical to performance of the Evolutionary Algorithm. The Monte Carlo simulation, however, performs slightly worse than the other probabilistic approaches.

Although the results are nearly the same when considering only 1500 fitness evaluations, the Evolutionary Algorithm (as well as Monte Carlo and Hill Climbing) cannot compete with APGAN or RPMC concerning run-time performance. E.g., APGAN needs less than 2.3 second for all graphs on a SUN SPARC 20, while the run-time of the Evolutionary Algorithm varies from 0.1 seconds up to 5 minutes (3000 fitness evaluations).

\footnotetext{
${ }^{1}$ Laplacian pyramid (minimal buffer cost: 99); QMF filterbank, one-sided tree (minimal buffer cost: 108).
} 


\begin{tabular}{|l|l|l|l|l|l|l|l|}
\hline System & BMLB & APGAN & RPMC & MC & HC & EA & $\begin{array}{l}\text { EA + } \\
\text { APGAN }\end{array}$ \\
\hline \hline $\begin{array}{l}\text { Fractional } \\
\text { decimation }\end{array}$ & 47 & 47 & 52 & 47 & 47 & 47 & 47 \\
\hline $\begin{array}{l}\text { Laplacian } \\
\text { pyramid }\end{array}$ & 95 & 99 & 99 & 99 & 99 & 99 & 99 \\
\hline $\begin{array}{l}\text { Nonuniform } \\
\text { filterbank } \\
\text { (1/3, } 2 / 3 \text { splits }) \\
(4 \text { channels })\end{array}$ & 85 & 137 & 128 & 143 & 126 & 126 & 126 \\
\hline $\begin{array}{l}\text { Nonuniform } \\
\text { filterbank } \\
\begin{array}{l}(1 / 3,2 / 3 \text { splits }) \\
\text { (6 channels) }\end{array}\end{array}$ & 224 & 756 & 589 & 807 & 570 & 570 & 570 \\
\hline $\begin{array}{l}\text { QMF } \\
\text { nonuniform- } \\
\text { tree filterbank }\end{array}$ & 154 & 160 & 171 & 165 & 160 & 160 & 159 \\
\hline $\begin{array}{l}\text { QMF filterbank } \\
\text { (one-sided tree) }\end{array}$ & 102 & 108 & 110 & 110 & 108 & 108 & 108 \\
\hline $\begin{array}{l}\text { QMF analysis } \\
\text { only }\end{array}$ & 35 & 35 & 35 & 35 & 35 & 35 & 35 \\
\hline $\begin{array}{l}\text { QMF tree } \\
\text { filterbank } \\
\text { (4 channels })\end{array}$ & 46 & 46 & 55 & 46 & 47 & 46 & 46 \\
\hline $\begin{array}{l}\text { QMF tree } \\
\text { filterbank } \\
(8 \text { channels })\end{array}$ & 78 & 78 & 87 & 78 & 80 & 80 & 78 \\
\hline $\begin{array}{l}\text { QMF tree } \\
\text { filterbank } \\
\text { (16 channels })\end{array}$ & 166 & 166 & 200 & 188 & 190 & 197 & 166 \\
\hline satellite receiver & 1540 & 1542 & 2480 & 1542 & 1542 & 1542 & 1542 \\
\hline
\end{tabular}

Table 4.1: Comparison of performance on practical examples; the probabilistic algorithms stopped after 3000 fitness evaluations.

The results concerning the random graphs are summarized in Table 4.2; again, the stochastic approaches were aborted after 3000 fitness evaluations. ${ }^{2}$ Interestingly, for these graphs APGAN only in $15 \%$ of all cases is better than Monte Carlo and only on in two cases better than the Evolutionary Algorithm. On the other hand, it is outperformed by the Evolutionary Algorithm $99 \%$ of the time. ${ }^{3}$ This is almost identical to the comparison between Hill Climbing and APGAN. As RPMC is known to be better suited for irregular graphs than APGAN [BML96], its better performance $(65.5 \%)$ is not surprising when directly compared to APGAN. Although, it is beaten by the Evolutionary Algorithm as well as Hill Climbing in 95.5\% and 96.5\% of the time, respectively. These results are very promising, but have to be considered in association with their quality, i.e., the magnitude of the buffer costs achieved.

Thus, we investigate the issue of deviation in the results on the random graphs

\footnotetext{
${ }^{2}$ The Evolutionary Algorithm ran about 9 minutes on each graph, the time for running APGAN was constantly less than 3 seconds.

${ }^{3}$ Considering 1500 fitness calculations, this percentage decreases only minimally to $97.5 \%$.
} 


\begin{tabular}{|l||l|l|l|l|l|l|}
\hline \multicolumn{1}{|c||}{} & APGAN & RPMC & MC & HC & EA & $\begin{array}{l}\text { EA }+ \\
\text { APGAN }\end{array}$ \\
\hline \hline APGAN & $0 \%$ & $34.5 \%$ & $15 \%$ & $0 \%$ & $1 \%$ & $0 \%$ \\
\hline RPMC & $65.5 \%$ & $0 \%$ & $29.5 \%$ & $3.5 \%$ & $4.5 \%$ & $2.5 \%$ \\
\hline MC & $85 \%$ & $70.5 \%$ & $0 \%$ & $0.5 \%$ & $0.5 \%$ & $1 \%$ \\
\hline HC & $100 \%$ & $96.5 \%$ & $99.5 \%$ & $0 \%$ & $70 \%$ & $57 \%$ \\
\hline EA & $99 \%$ & $95.5 \%$ & $99.5 \%$ & $22 \%$ & $0 \%$ & $39 \%$ \\
\hline EA + APGAN & $100 \%$ & $97.5 \%$ & $99 \%$ & $32.5 \%$ & $53.5 \%$ & $0 \%$ \\
\hline
\end{tabular}

Table 4.2: Comparison of performance on 200 50-actor SDF graphs (3000 fitness evaluations); for each row the numbers represent the fraction of random graphs on which the correspondig heuristic outperforms the other approaches.

in the following sections. Section 4.1 compares the Evolutionary Algorithm to APGAN and examines, whether a combination of both approaches improves the overall performance. In Section 4.2 we analyze the outcomes produced by the Evolutionary Algorithm regarding RPMC. Finally, the remaining two sections compare the Evolutionary Algorithm to the other probabilistic optimization methods, Monte Carlo and Hill Climbing, respectively.

\subsection{Comparing the Evolutionary Algorithm to APGAN}

Figure 4.1 gives an impression of the quality of the results (magnitude of the buffer costs) computed by the Evolutionary Algorithm relative to APGAN. The left hand side shows the deviations when aborting the Evolutionary Algorithm after 1500 fitness evaluations, the right hand side shows the same for 3000 fitness evaluations; for both cases a complete plot as well as a reduced plot focusing on relevant details is given. The $x$ axis ranges from 1 to 200 representing the various random graphs, while the $y$ axis denotes the factor by which the Evolutionary Algorithm is superior (positive range) or inferior (negative range), respectively, to APGAN. E.g., the $y$ value $200 \%$ means that the buffer cost yielded by the Evolutionary Algorithm is half the APGAN result on the same random graph; on the other hand, the $y$ value $-200 \%$ indicates that APGAN performs twice as good as the Evolutionary Algorithm on a particular graph.

Together with the results presented in Table 4.2, the superiority of the Evolutionary Algorithm, regarding highly irregular graphs, becomes evident. The buffer costs are half the costs computed by APGAN in average. A deviation up to the factor 28 is achieved on a particular graph, in six cases an improvement by a factor greater than 5 can be observed. As stated in [BML97], APGAN performs well on graphs that have relatively regular topological structures and rate changes. Since large random graphs are rather expected to be irregular, this may explain the bad performance of APGAN relative to the Evolutionary Algorithm on our collection of 


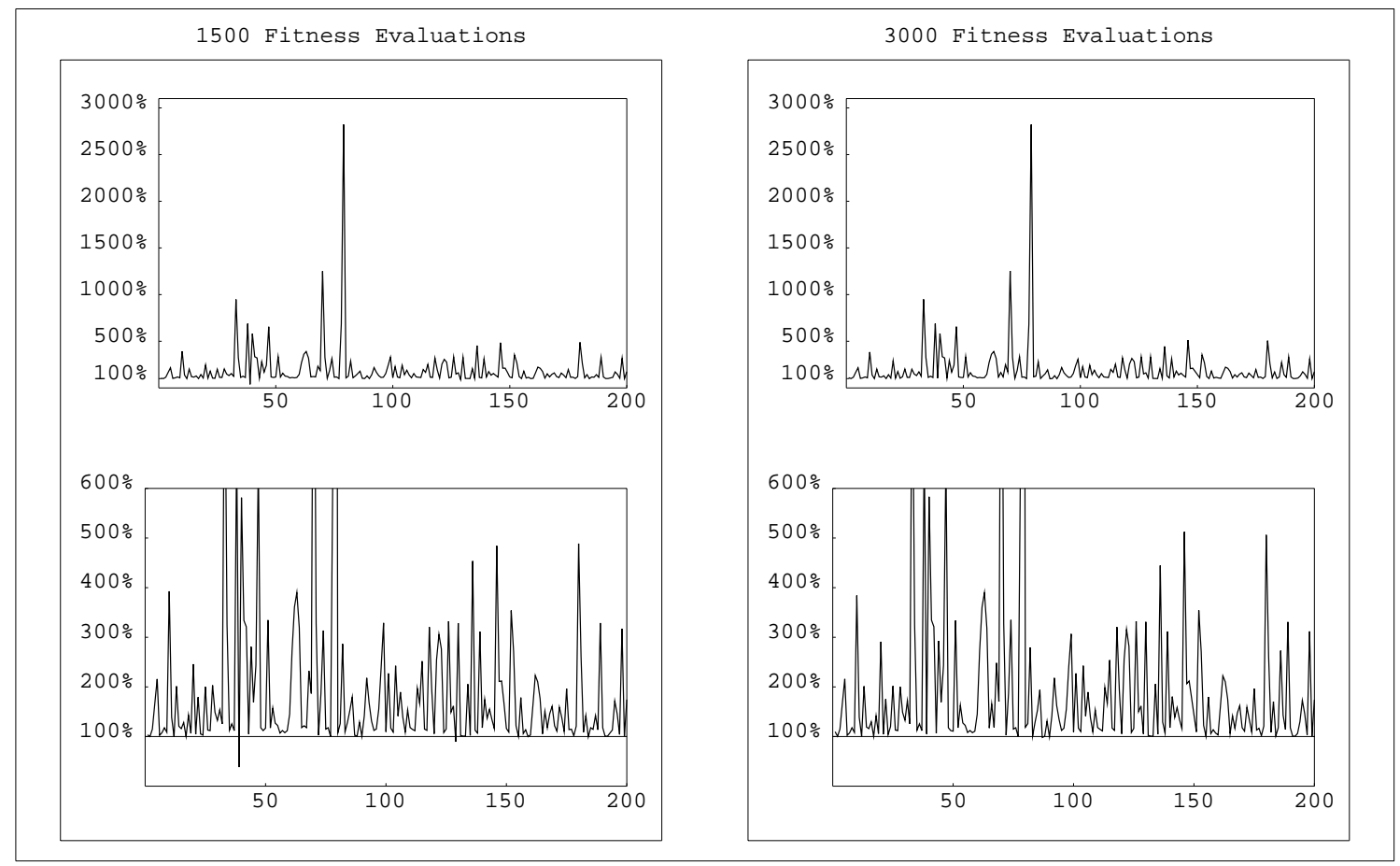

Figure 4.1: Quality of the results produced by the Evolutionary Algorithm relative to APGAN. The two upper graphics show all points in one plot, while the lower two zoom into interesting regions.

random graphs.

Inserting the APGAN solution into the initial population seems to have only slight influence on the quality of results (cf. Figure 4.2). After 3000 fitness calculations the average buffer cost are smaller by a factor of 1.0007 which is rather caused by random effects than due to better performance. On the other hand, regarding the case of 1500 fitness evaluations, the extension of the Evolutionary Algorithm leads to an improvement of a factor 1.039. This might indicate that this extension converges slightly faster towards the end result. We examined that issue on three random graphs, comparing the performance of both approaches in dependence on the number of fitness evaluations (see Figure 4.6 on page 28). With respect to one particular graph, this hypothesis could be confirmed, while on the other two graphs we found no significant differences in the behavior.

Nevertheless, the Evolutionary Algorithm and APGAN complement one another when regarding both regular and irregular graphs. On the one hand, APGAN exploits regularity that arises commonly in practical applications, and is provably optimal for an interesting class of graphs ([BML96]). On the other hand, the run-time needed by APGAN can be neglected in comparison to the Evolutionary Algorithm. Hence, it is a good idea to combine the two approaches in the presented manner. Table 4.2 shows that $53.5 \%$ of the time smaller buffer costs can be achieved, while the Evolutionary Algorithm without APGAN outperforms the combined approach 


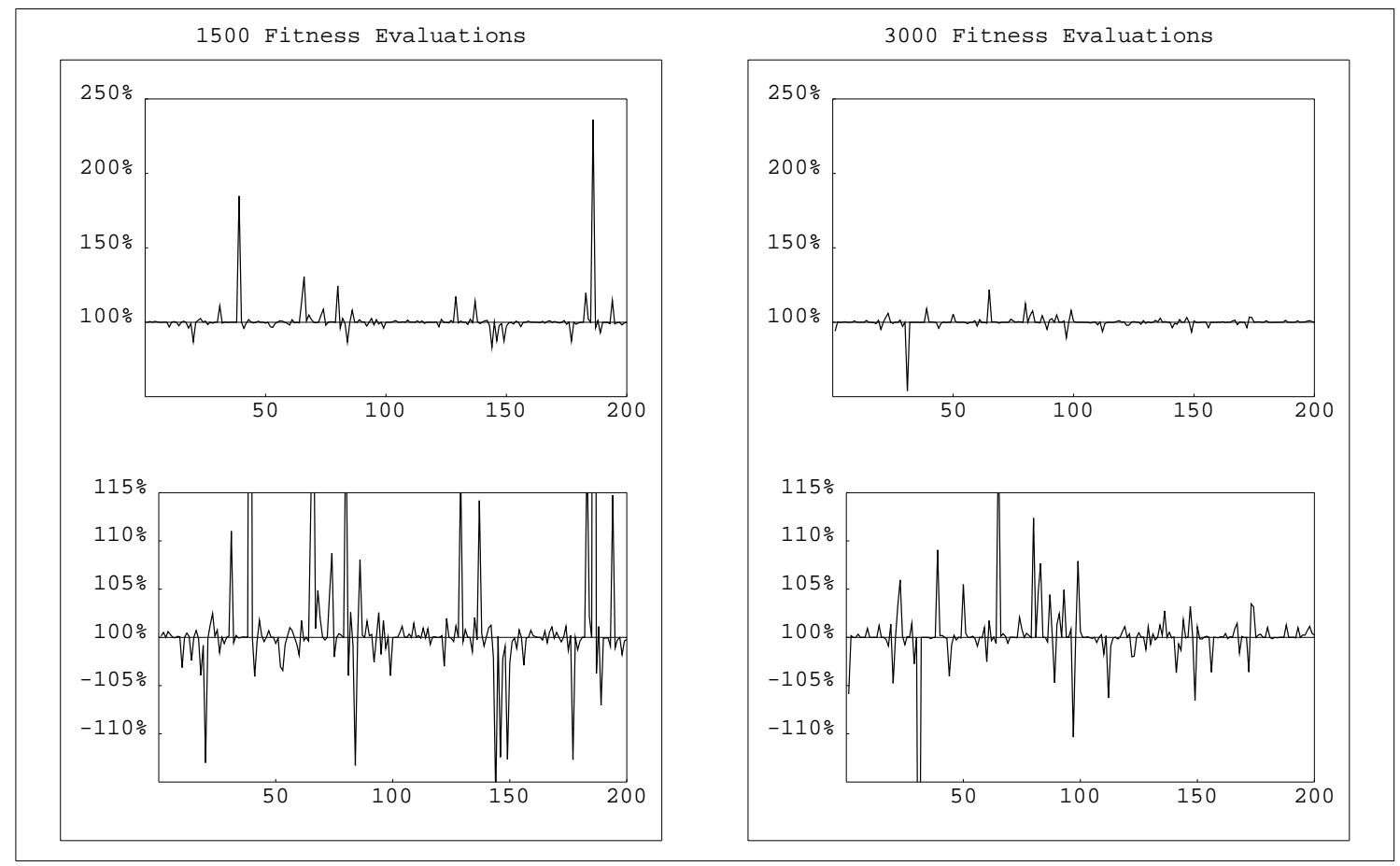

Figure 4.2: Influence of inserting the APGAN solution into the initial population on the quality of results.

in only $39 \%$ of all cases.

\subsection{Comparing the Evolutionary Algorithm to RPMC}

Relative to APGAN, RPMC is expected to perform well on graphs that have rather irregular rate changes and irregular topologies [BML96]; although, it has no known optimality property relevant to practical graphs. Therefore, the question is whether RPMC or the Evolutionary Algorithm is the more appropriate complement to APGAN.

Table 4.2 shows that RPMC is outperformed by the Evolutionary Algorithm in $95.5 \%$ of the time (3000 fitness calculations ${ }^{4}$ ). Moreover, the buffer costs achieved by RPMC are significantly greater than the ones computed by the Evolutionary Algorithm (cf. Figure 4.3). In average the results are worse by a factor of 1.58 and 1.6 in the case of 1500 and 3000 fitness evaluations respectively. In one case the Evolutionary Algorithm performs better by a factor of 10; on twelve random graphs this factor is greater than 3 .

These results indicate that the Evolutionary Algorithm is superior to RPMC on highly irregular graphs. Additionally, it performs also better on the practical graphs

\footnotetext{
${ }^{4} 94 \%$ in the case of 1500 fitness calculations.
} 


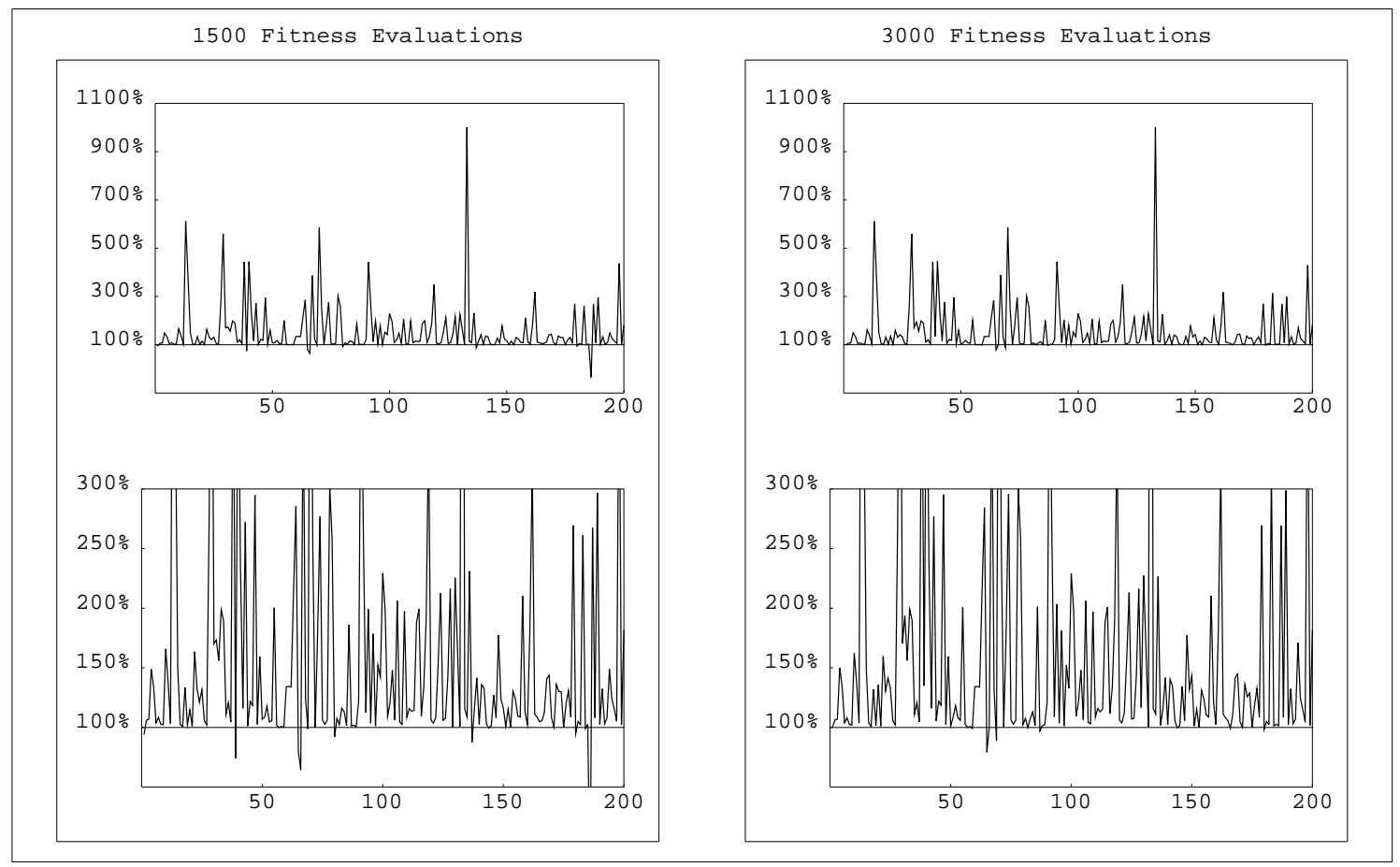

Figure 4.3: Quality of the results produced by the Evolutionary Algorithm relative to the ones achieved by RPMC.

listed in Table 4.1. Therefore, the Evolutionary Algorithm is the better choice when extra execution time is tolerable.

We also thought of incorporating RPMC into the Evolutionary Algorithm (similar to APGAN). However, since RPMC does not have any optimality property and surpasses the combination of Evolutionary Algorithm and APGAN in only $2.5 \%$ of the time (cf. Table 4.2), we did no further investigations in this direction. Nevertheless, one might combine APGAN, RPMC and Evolutionary Algorithm as the run-time of APGAN and RPMC can be neglected in comparison to the evolutionary approach.

\subsection{Evolutionary Algorithm versus Monte Carlo}

The Monte Caro implementation is very simple. Based an a uniform probability distribution, a certain number of topological sorts (bounded by the maximum number of fitness calculations) is generated randomly and to each of them GDPPO is applied. Finally, the topological sort with minimal buffer cost among all considered is the outcome of the algorithm. Since no mutation and crossover are necessary, this algorithm runs slightly faster than the Evolutionary Algorithm. 


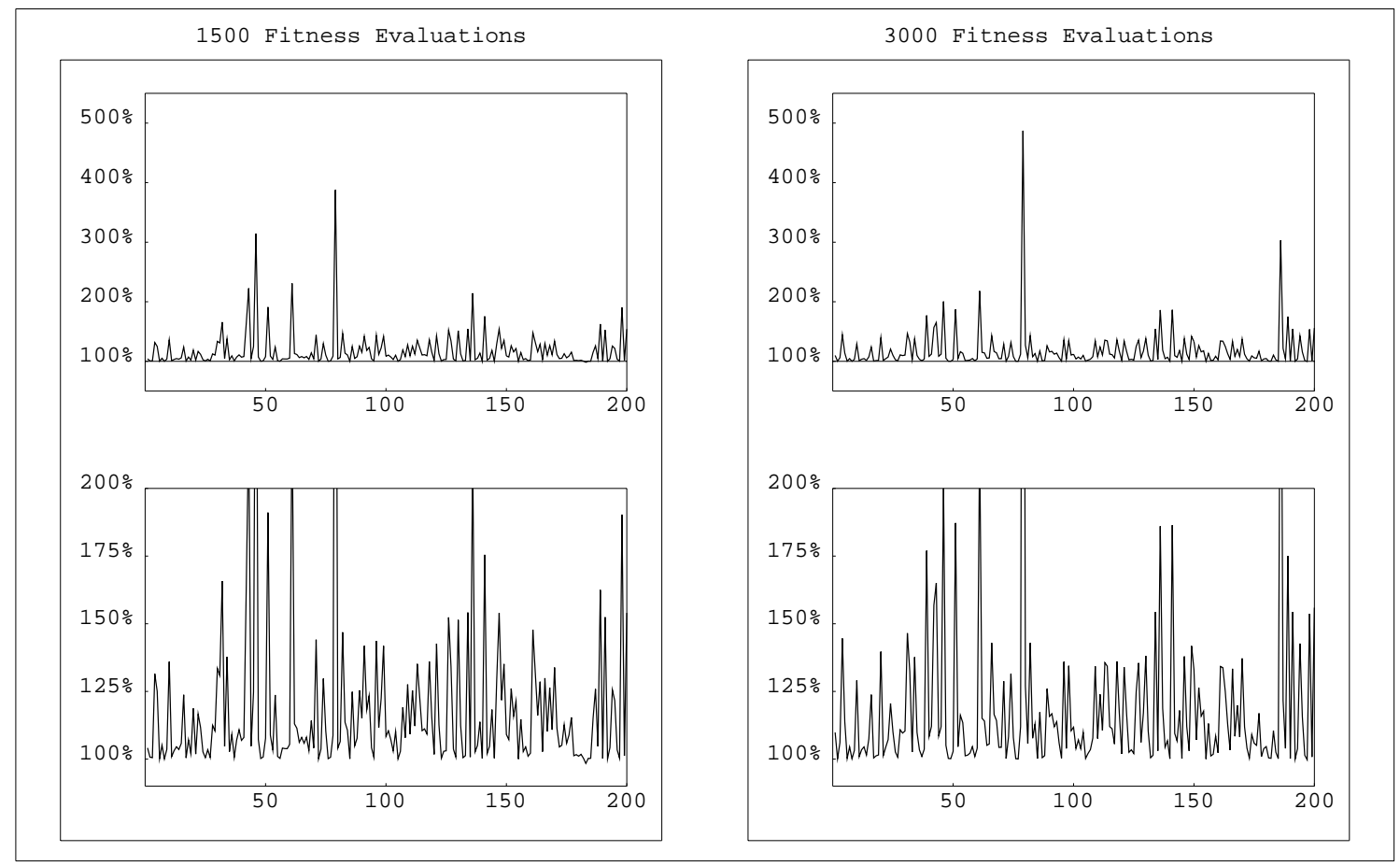

Figure 4.4: Quality of the results produced by the Evolutionary Algorithm relative to the Monte Carlo method.

Disregarding the quality of results, the Evolutionary Algorithm clearly outperforms Monte Carlo (99\% of the time, cf. Table 4.2), while there is only one case where Monte Carlo yields a better result. This is also true when considering the deviation of results, as can be seen in Figure 4.4. In average, the buffer costs computed by the Evolutionary Algorithm are only a fraction of 0.84 of the costs produced by the Monte Carlo heuristic. The case of 1500 fitness evaluations looks identical.

These results are substantiated by the curves shown in Figure 4.6 on page 28. With it, we considered three random graphs and took the mean fitness value of ten independent runs at each point in time. Monte Carlo performs worse than the Evolutionary Algorithm as well as Hill Climbing.

In summary it may be said that the Evolutionary Algorithm is far better suited for this application domain than the Monte Carlo approach - in contrast to the presumption we made in Section 3.3 that the opposite may be true.

\subsection{Evolutionary Algorithm versus Hill Climbing}

We implemented Hill Climbing similarly to the Evolutionary Algorithm. First a starting point $p$, representing a permutation over the graph nodes, is chosen at random. Then the following loop is executed $n-1$ times, where $n$ denotes the maximum number of fitness evaluations: 


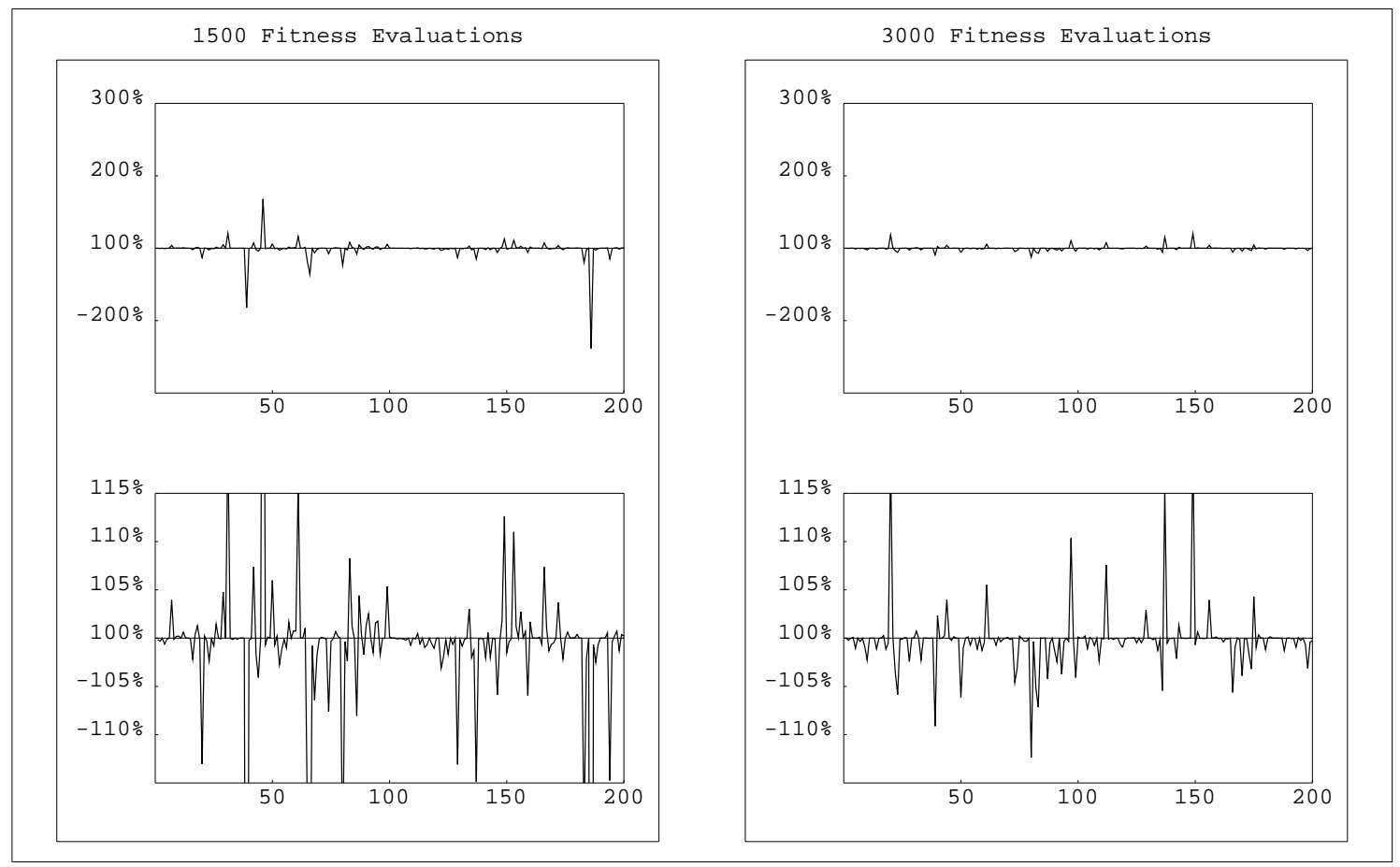

Figure 4.5: Quality of the results produced by the Evolutionary Algorithm relative to the Hill Climbing method.

1. A new point $p^{\prime}$ is created by appling the scramble list mutation operator to $p$ (cf. Section 3.2).

2. Both $p$ and $p^{\prime}$ are transformed into a topological sort by means of the repair mechanism described in Section 3.1.

3. The resulting topological sorts are evaluated by the SDF framework; if the phenotype of $p^{\prime}$ requires less buffer memory than the phenotype of $p, p$ is set to $p^{\prime}$.

Compared to the Evolutionary Algorithm, the results listed in Table 4.2 seem to indicate a superiority of the Hill Climbing method; its results are better $70 \%$ of the time and worse only $22 \%$ of the time. But when we examined the variations of the buffer costs computed by the two algorithms, we found no reliable indication for this hypothesis.

Figure 4.5 shows the quality of the results calculated by the Evolutionary Algorithm relative to the ones achieved by Hill Climbing. In average, the performance of Hill Climbing is better by a factor of 1.0019 - a non-signficant margin.

Also the curves plotted in Figure 4.6 do not provide evidence of the superiority or the inferiority of one approach. Further investigations are necessary to answer this question. 

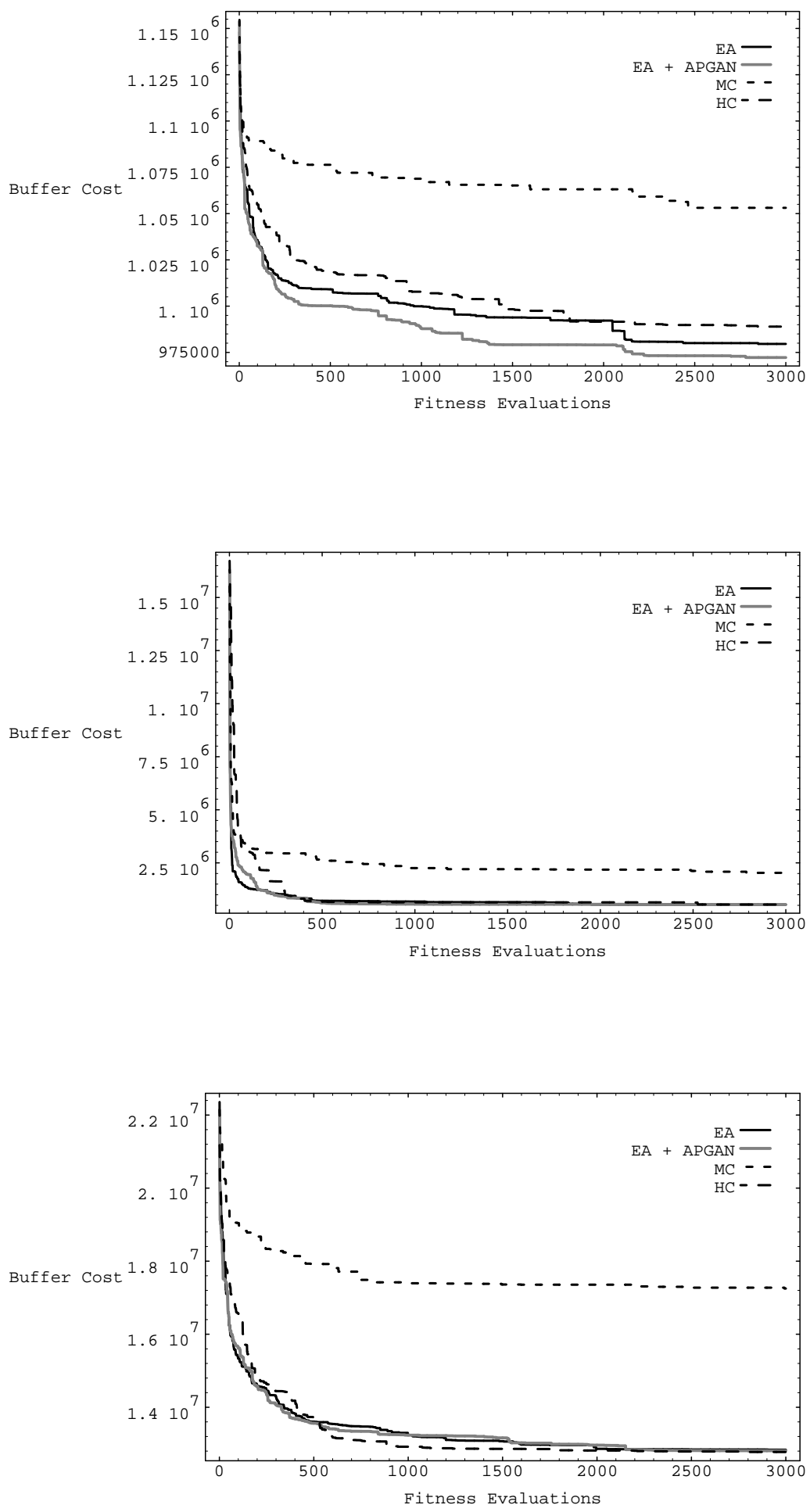

Figure 4.6: Performance of the probabilistic methods on three different random graphs. For both each graph and each method we took the average buffer cost over ten independent runs. 


\section{Chapter 5}

\section{Summary and Conclusions}

We have proposed a new approach for finding buffer-optimal schedules among the set of program-memory schedules for uni-processor implementations of SDF graphs using a two-step approach: An Evolutionary Algorithm (EA) is used to explore the search space of legal actor firing orders. During the fitness computation, a $\mathcal{O}\left(N^{3}\right)$ dynamic programming post-optimizer calculates a loop nest with minimum buffer cost for the given actor firing order.

The results obtained have shown that this approach, though being more computationally expensive than existing algorithms such as PGAN and RPMC, promise to find better solutions in a reasonable amount of run-time. The differences in quality seem to become even more severe for larger graphs with irregular structures. Here, we tested the performance only for graphs with up to 50 nodes.

The experiments have also shown that Hill Climbing could be a good alternative to the EA approach. However, in this paper, we did not investigate this further.

The ease to define and change the fitness function when using an Evolutionary Algorithm makes us think of the following extensions as part of the continuation of this preliminary investigation:

- Exploitation of different buffer allocation schemes:

The buffer cost model used in our experiments so far considered only statically allocated contigous memory segments that were not shared between different arc buffers. However, it should be possible to consider also memory-buffer sharing and more complex types of memory allocation. This should be possible be considering slightly more complex fitness functions. In this case, the optimization procedure does not only determine the optimum buffer costs but also makes decisions of what buffer organization to choose for each arc of a given graph.

- Exploitation of optimal flat single appearance schedules:

The generated topological sorts could be considered directly as flat single appearance schedules. For these, the dynamic programming post-optimizer could be replaced by a memory allocation algorithm that exploits sharing between different arcs. Because this problem is NP-hard, too (for non-unit buffer-sizes), 
we are currently developing efficient buffer allocation heuristics based on the life-time of memory blocks.

- Consideration of general (non-acyclic) SDF graphs:

Here, we considered only acyclic SDF graphs. This had the advantage that the coding of actors had a linear space-complexity in terms of the number of nodes in the graph. This low complexity, however, was obtained at the cost of only exploiting buffer-memory schedules at the front of constant programmemory (single appearance schedules). Questions such as what is a minimal program-memory for designs at the front of buffer-memory optimal schedules could not be answered. Note, however, that in case of multiple-appearance schedules, the consideration of an amount of actor firings that is given by the number of entries in the minimal repetition vector seems to be unavoidable. Whether this approach is not prohibitive for typical DSP algorithms, however, will first have to be shown.

Finally, not only these two criterion are of importance in the generation of DSP implementations of SDF graphs, but also other criterion such as execution time, and throughput, respectively: It would be interesting to investigate trade-offs between code-inlining and subprogram generation so to trade between program memory and execution time. In particular, code-inlining might still not be favorable in case of SOS (system on a chip) implementations of DSP algorithms. There, subprogram generation may lead to a dramatic decrease of required program memory in case multiple-appearance schedules are investigated. Whether EAs will help to answer these questions will be a project of longer terms. 


\section{Bibliography}

[ASU86] A. V. Aho, R. Sethi, and J. D. Ullman. Principles of Compiler Design. Addison-Wesley, Reading, MA, 1986.

[BBHL95] S. Bhattacharyya, J. T. Buck, S. Ha, and E. A. Lee. Generating compact code from dataflow specifications of multirate signal processing specifications. IEEE Trans. on Circuits and Systems - I: Fundamental Theory and Applications, 42(3):138-150, March 1995.

[Bha94] S. Bhattacharyya. Compiling data flow programs for digital signal processing. Technical Report UCB/ERL M94/52, Electronics Research Laboratory, UC Berkeley, July 1994.

[BHLM91] J. Buck, S. Ha, E.A. Lee, and D.G. Messerschmitt. Ptolemy: A framework for simulating and prototyping heterogeneous systems. International Journal on Computer Simulation, 4:155-182, 1991.

[BL94] S. Bhattacharyya and E. A. Lee. Looped schedules for dataflow descriptions of multirate signal processing algorithms. Journal of Formal Methods in System Design, 5(3), December 1994.

[BML96] S. S. Bhattacharyya, P. K. Murthy, and E. A. Lee. Software Synthesis from Dataflow Graphs. Kluwer Academic Publishers, Norwell, MA, 1996.

[BML97] S. S. Bhattacharyya, P. K. Murthy, and E. A. Lee. APGAN and RPMC: Complementary heuristics for translating DSP block diagrams into efficient software implementations. J. Design Automation for Embedded Systems, January 1997.

[Dav91] Lawrence Davis. Handbook of Genetic Algorithms, chapter 6, pages 7290. Van Nostrand Reinhold, New York, 1991.

[dlMT93] Michael de la Maza and Bruce Tidor. An analysis of selection procedures with particular attention paid to proportional and boltzmann selection. In Stephanie Forrest, editor, Proceedings of the Fifth International Conference on Genetic Algorithms, pages 124-131, San Mateo, California, 1993. Morgan Kaufmann. 
[DP96] T. C. Denk and K. K. Parhi. Lower bounds on memory requirements for statically scheduled dsp programs. J. of VLSI Signal Processing, pages 247-264, 1996.

[Fab82] J. Fabri. Automatic Storage Optimization. UMI Research Press, 1982.

[FM91] B. R. Fox and M. B. McMahon. Genetic operators for sequencing problems. In Gregory J. E. Rawlins, editor, Foundations of Genetic Algorithms, pages 284-300. Morgan Kaufmann, San Mateo, California, 1991.

[GJ79] M.R. Garey and D.S. Johnson. Computers and Intractability: A Guide to the Theory of NP-Completeness. Freeman, New York, 1979.

[Gol89] David E. Goldberg. Genetic Algorithms in Search, Optimization, and Machine Learning. Addison-Wesley, Reading, Massachusetts, 1989.

[Hsu87] W.-C. Hsu. Register allocation and code scheduling for load/store architectures. Technical report, Department of Computer Science, University of Wisconsin at Madison, 1987.

[KNDK96] D. J. Kolson, A. N. Nicolau, N. Dutt, and K. Kennedy. Optimal register assignment to loops for embedded code generation. ACM Trans. on Design Automation of Electronic Systems, 1(2):251-279, 1996.

[Koz92] John R. Koza. Genetic Programming: On the Programming of Computers by Means of Natural Selection. MIT Press, Cambridge, Massachusetts, 1992.

$\left[\mathrm{LEP}^{+}\right.$90] R. Lauwereins, M. Engels, J. A. Peperstraete, E. Steegmans, and J. Van Ginderdeuren. Grape: A CASE tool for digital signal parallel processing. IEEE ASSP Magazine, 7(2):32-43, April 1990.

[LM87] E.A. Lee and D.G. Messerschmitt. Synchronous dataflow. Proceedings of the IEEE, 75(9):1235-1245, 1987.

[MBL94] P. K. Murthy, S. S. Bhattacharyya, and E. A. Lee. Minimizing memory requirements for chain-structured Synchronous Data Flow Programs. In Proc. of ICASSP-94, Adelaide, Australia, 1994.

[Me95] P. Marwedel and G. Goossens (eds.). Code generation for embedded processors. Kluwer Academic Publishers, Norwell, MA, 1995.

[RPM92] S. Ritz, M. Pankert, and H. Meyr. High level software synthesis for signal processing systems. In Proc. Int. Conf. on Application-Specific Array Processors, pages 679-693, Berkeley, CA, 1992.

[RWM95] S. Ritz, M. Willems, and H. Meyr. Scheduling for optimum data memory compaction in block diagram oriented software synthesis. In Proceedings of the International Conference on Acoustics, Speech and Signal Processing, volume 4, pages 2651-2654, May 1995. 